\title{
Vinyl-Stilbene Inhibits Human Norovirus RNA Replication by Activating Heat-Shock Factor-1
}

\author{
Ahrim Lee ${ }^{1, \dagger}$, Jieun Sung ${ }^{1, \dagger}$, Dipesh S. Harmalkar ${ }^{1,2, \dagger}$, Hyeseul Kang ${ }^{1}$, Hwayoung Lee ${ }^{1}$, Kyeong Lee $^{1, *}$ and \\ Choongho Lee ${ }^{1, *}$
}

${ }^{1}$ College of Pharmacy, Dongguk University-Seoul, Goyang 10326, Republic of Korea

${ }^{2}$ Department of Chemistry, Dnyanprassarak Mandal's College \& Research Centre, Bardez, Goa 403507, India

\begin{abstract}
Norovirus (NV) is the most common cause of viral gastroenteritis, with the potential to develop into a fatal disease in those who are immuno-compromised, and effective vaccines and treatments are still non-existent. In this study, we aimed to elucidate the molecular mechanism of the previously identified NV replication inhibitor utilizing a vinyl-stilbene backbone, AC-1858. First, we confirmed the inhibition of the NV RNA replication by a structural analog of AC-1858, AC-2288 with its exclusive cytoplasmic subcellular localization. We further validated the induction of one specific host factor, the phosphorylated form of heat shock factor (HSF)-1, and its increased nuclear localization by AC-1858 treatment. Finally, we verified the positive and negative impact of the siRNA-mediated downregulation and lentivirus-mediated overexpression of HSF-1 on NV RNA replication. In conclusion, these data suggest the restrictive role of the host factor HSF-1 in overall viral RNA genome replication during the NV life cycle.
\end{abstract}

Key Words: Norovirus, Vinyl-stilbene, RNA replication inhibitor, Heat shock factor-1, Antiviral mechanism of action

\section{INTRODUCTION}

Norovirus (NV) is best known as the most common cause of viral gastroenteritis (Glass et al., 2009). Human NV is a nonenveloped virus of the Caliciviridae family with a single-stranded positive-sense RNA genome (Morillo and Timenetsky, 2011). NVs are genetically classified into seven different genogroups (GI, GII, GIII, GIV, GV, GVI, and GVII) based on their sequence similarities (Atmar et al., 2019). In particular, genogroups $\mathrm{Gl}$ and GIl represent most NVs with a host tropism for humans (Vinje et al., 2000). In general, the NV genome is composed of three open reading frames (ORF1-3). ORF1 encodes a large $200-k D a$ polyprotein whose cleavage by the virally encoded protease NS6 leads to the production of six nonstructural proteins. Among these nonstructural proteins, the viral protease NS6 and RNA-dependent RNA polymerase NS7 have been attractive targets for NV-specific drug discovery efforts due to their virus-specific enzymatic activities (Hardy, 2005; Thorne and Goodfellow, 2014).

NVs are very stable in the external environment. Most NV infections are generally caused by the viral contamination of food and water (de Graaf et al., 2016), and transmission occurs through the fecal-oral route (Atmar and Estes, 2006). The symptoms of NV infection include fever, headache, abdominal pain, non-bloody diarrhea, and vomiting, the latter of which can lead to a sore throat (Turcios et al., 2006). The average incubation period of NV infection is around $12 \mathrm{~h}$ to $48 \mathrm{~h}$. It can cause chronic diarrhea with a high mortality rate, especially in immunocompromised patients, the elderly, and young children (Bok and Green, 2012). Unfortunately, there are no effective vaccines or treatments available for NV infections so far. According to the Centers for Disease Control and Prevention (CDC), NV causes about 200,000 deaths per year worldwide and is estimated to cost $\$ 60$ billion each year due to increased health care costs and reduced productivity (https://www.cdc. gov/norovirus/index.html).

Historically, the in vitro culture of NV has been extremely challenging due to its complex requirement for efficient ex-vivo growth. Therefore, a murine NV has been frequently used as a surrogate model to study NV (Wobus et al., 2006). As another

\section{Open Access https://doi.org/10.4062/biomolther.2021.153}

This is an Open Access article distributed under the terms of the Creative Commons Attribution Non-Commercial License (http://creativecommons.org/licenses/by-nc/4.0/) which permits unrestricted non-commercial use, distribution, and reproduction in any medium, provided the original work is properly cited.
Received Sep 28, 2021 Revised Oct 19, 2021 Accepted Oct 25, 2021 Published Online Nov 16, 2021

\section{*Corresponding Authors}

E-mail: kaylee@dongguk.edu (Lee K), choongholee@dongguk.edu (Lee C) Tel: +82-31-961-5214 (Lee K), +82-31-961-5223 (Lee C)

Fax: +82-31-961-5206 (Lee K), +82-31-961-5206 (Lee C)

${ }^{\dagger}$ The first three authors contributed equally to this work. 
complementing research tool, a human NV replicon cell line (HG23 cells) was developed for drug-screening (Lee and Ko, 2016). This cell line, which was derived from human hepatoma 7.5 cells, maintains the human Norwalk NV replicon RNA of $\mathrm{GI}$. Due to the insertion of a neomycin phosphotransferase gene into the viral genome, only cells with a high copy number of NV RNAs survive in the presence of selective antibiotic pressure (Chang et al., 2006). In addition, the expression of a neomycin phosphotransferase can be used as an indirect marker for the quantification of the viral RNA genome replication.

Previously, our laboratory has screened in-house library compounds with a variety of chemical moieties to discover therapeutic candidates for NV infection using HG23 cells. In this study, we identified a group of chemicals with a stilbenoid structure and found them to be NV RNA replication inhibitors with little cytotoxicity. Three rounds of structure- and activityrelationship experiments further disclosed AC-1858 ((E)-(4(3,5-dimethoxy-4-vinylstyryl)phenyl)(4-(4-(trifluoromethyl) benzyl)piperazin-1-yl)methanone) as the most potent anti-NV replication inhibitor (Harmalkar et al., 2019). In addition, subsequent PCR array analysis suggested the potential role of a host factor called heat shock factor-1 (HSF-1) in the pharmacological inhibition of NV RNA replication by AC-1858 (Harmalkar et al., 2019). However, the detailed molecular mechanism of AC-1858-mediated suppression of NV RNA replication through the modulation of HSF-1 was not clear. In this study, we sought to elucidate the molecular function of the newly discovered antiviral host factor HSF-1 in the context of the negative modulation of NV RNA replication. For this goal, we utilized the newly synthesized molecular probes for AC-1858 as well as techniques for siRNA-mediated downregulation and lentivirus-mediated overexpression of HSF-1 to further characterize the potential restrictive role of HSF-1 in the regulation of NV RNA replication during the NV life cycle.

\section{MATERIALS AND METHODS}

\section{General chemistry}

All the commercial chemicals were of reagent grade and were used without further purification. Solvents were dried with standard procedures. All the reactions were carried out under an atmosphere of dried argon in flame-dried glassware. The proton nuclear magnetic resonance ( $\left.{ }^{1} \mathrm{H}-\mathrm{NMR}\right)$ spectra were determined on a Varian $(400 \mathrm{MHz})$ spectrometer (Varian Medical Systems, Inc., Palo Alto, CA, USA), and ${ }^{13} \mathrm{C}-\mathrm{NMR}$ spectra were recorded on a Varian $(100 \mathrm{MHz})$ spectrometer. Multiplicities of NMR signals were reported using different abbreviations like singlet (s), doublet (d), triplet (t), quartet (q), and multiplet $(\mathrm{m})$. The downfield chemical shifts are provided in parts per million (ppm) with coupling constants in hertz $(\mathrm{Hz})$. The mass spectra were recorded using high-resolution mass spectrometry (HRMS) (electron ionization MS) obtained on a JMS-700 mass spectrometer (Jeol, Tokyo, Japan) or using HRMS (electrospray ionization MS) obtained on a G2 QTOF mass spectrometer. The products from all the reactions were purified by flash column chromatography using silica gel 60 (230-400 nm mesh, Kieselgel 60). Additionally, thin-layer chromatography on $0.25-\mathrm{mm}$ silica plates (Merck, Kenilworth, NJ, USA; silica gel 60 F254) was used to monitor the reactions. Final product purity was checked by reversed-phase high-pres- sure liquid chromatography (RP-HPLC) performed on a Waters Corp (Milford, MA, USA). An HPLC system equipped with an ultraviolet detector was set to $254 \mathrm{~nm}$. The mobile phases used were: (A) $\mathrm{H}_{2} \mathrm{O}$ containing $0.05 \%$ trifluoroacetic acid and (B) $\mathrm{CH}_{3} \mathrm{CN}$. HPLC employed a YMC Hydrosphere C18 (HS302) column (5- $\mu \mathrm{m}$ particle size, $12-\mathrm{nm}$ pore size) that was $4.6 \mathrm{~mm}$ in diameter $\times 150 \mathrm{~mm}$ in length with a flow rate of 1.0 $\mathrm{mL} / \mathrm{min}$. Compound purity was assessed using $\mathrm{CH}_{3} \mathrm{CN}: 0.05 \%$ TFA in $\mathrm{H}_{2} \mathrm{O}$ at a 25:75 to $100: 0$ ratio. The purity of all biologically evaluated compounds was $>95 \%$.

\section{Synthesis of (E)-(4-(3,5-dimethoxy-4-vinylstyryl)phenyl) (4-(4-(trifluoromethyl)benzyl)piperazin-1-yl)methanone (AC-1858)}

Purification by flash column chromatography (silica gel, $0-70 \%$ EtOAc in hexanes) provided AC-1858 as a light-yellow solid $\left(51.0 \mathrm{mg}, 59 \%\right.$ yield). ${ }^{1} \mathrm{H}-\mathrm{NMR}\left(\mathrm{CdCl}_{3}, 400 \mathrm{MHz}\right) \delta 7.58$ (d, J=8.0 Hz, 2H), 7.54 (d, J=8.0 Hz, 2H), 7.46 (d, J=8.0 Hz, $2 \mathrm{H}), 7.41$ (d, J=8.4 Hz, 2H), 7.11 (d, J=16.4 Hz, 1H), 7.07 (d, $J=16.8 \mathrm{~Hz}, 1 \mathrm{H}), 6.97(\mathrm{dd}, J=18.0,12.0 \mathrm{~Hz}, 1 \mathrm{H}), 6.71(\mathrm{~s}, 2 \mathrm{H})$, 6.11 (dd, J=18.0, $2.4 \mathrm{~Hz}, 1 \mathrm{H}$ ), 5.45 (dd, J=12.0, $2.8 \mathrm{~Hz}, 1 \mathrm{H}$ ), 3.91 (s, 6H), $3.78(\mathrm{br}, 2 \mathrm{H}), 3.59(\mathrm{~s}, 2 \mathrm{H}), 3.50(\mathrm{br}, 2 \mathrm{H}), 2.49$ (br, 4H) ${ }^{13} \mathrm{C}-\mathrm{NMR}\left(\mathrm{CdCl}_{3}, 100 \mathrm{MHz}\right) \delta 170.0,158.7,141.9$, 138.6, 136.9, 134.7, 130.2, 129.7, 129.4, 129.1, 127.8, 127.7, $127.1,126.4,125.4-125.2,118.6,115.1,102.4,62.3,55.8$, 53.0, 52.9, 47.7, 42.2. The HRMS (ESI) $\mathrm{m} / \mathrm{z}$ was calculated for $\mathrm{C}_{31} \mathrm{H}_{32} \mathrm{~F}_{3} \mathrm{~N}_{2} \mathrm{O}_{3}[\mathrm{M}+\mathrm{H}]^{+} 537.2365$, and found 537.2372; the RP-HPLC purity was $96.6 \%$ at $254 \mathrm{~nm}\left(t_{R}=12.78 \mathrm{~min}\right)$. Detailed experimental procedures, synthesis scheme, and NMR spectra regarding this compound can be found in the supporting information.

\section{Synthesis of (E)-(4-(3,5-dimethoxy-4-vinylstyryl)-3- (prop-2-yn-1-yloxy)phenyl)(4-(4-(trifluoromethyl)benzyl) piperazin-1-yl)methanone (AC-2288)}

HATU (75.0 mg, $0.19 \mathrm{mmol})$ was added to a solution of appropriate acid $(60.0 \mathrm{mg}, 0.16 \mathrm{mmol})$, 1-(4-trifluoromethylbenzyl)piperazine $(48.0 \mathrm{mg}, 0.19 \mathrm{mmol})$, and DIPEA $(0.08 \mathrm{~mL}$, $0.49 \mathrm{mmol}$ ) in DMF, and the reaction mixture was stirred at room temperature until complete consumption of the starting material. The reaction was monitored by TLC, and the reaction mixture was quenched with water and extracted with EtOAc. The combined organic layer was washed with brine, dried over anhydrous $\mathrm{MgSO}_{4}$, and concentrated under vacuum. Purification by flash column chromatography (silica gel, $0 \%$ to $70 \%$ EtOAc in hexane) produced (E)-(4-(3,5-dimethoxy-4vinylstyryl)-3-(prop-2-yn-1-yloxy)phenyl)(4-(4-(trifluoromethyl) benzyl)piperazin-1-yl)methanone (compound 10) as a paleyellow solid (30.0 mg, 31\%). ${ }^{1} \mathrm{H} \mathrm{NMR}\left(\mathrm{CdCl}_{3}, 400 \mathrm{MHz}\right) \delta$-7.63 (d, J=8.0 Hz, 1H), 7.59 (d, J=8.0 Hz, 2H), 7.46 (d, J=8.0 Hz, $2 \mathrm{H}$ ), 7.41 (d, J=16.4 Hz, 1H), 7.11-7.05 (2H, merged), 7.09 (d, $J=16.8 \mathrm{~Hz}, 1 \mathrm{H}), 6.98(\mathrm{dd}, J=18.0,12.0 \mathrm{~Hz}, 1 \mathrm{H}), 6.72(\mathrm{~s}, 2 \mathrm{H})$, 6.10 (dd, J=18.0, $2.8 \mathrm{~Hz}, 1 \mathrm{H}$ ), 5.44 (dd, J=12.2, $2.6 \mathrm{~Hz}, 1 \mathrm{H}$ ), 4.81 (d, J=2.0 Hz, 2H), 3.90 (s, 6H), 3.79 (br, 2H), 3.59 (s, 2H), 3.52 (s, 2H), 2.53 (s, 1H), 2.44 (br, 4H); ${ }^{13} \mathrm{C} \mathrm{NMR}\left(\mathrm{CdCl}_{3}, 100\right.$ $\mathrm{MHz}$ ) were $\delta 169.75,158.69,154.56,141.89,137.44,135.49$, $130.88,129.13,128.51,127.19,126.52,125.33,125.30$, $122.44,120.54,118.52,114.87,111.80,102.54,78.26,76.04$, $62.29,56.34,55.81,53.40,52.74,47.78,42.20$. The HRMS (ESI) $\mathrm{m} / \mathrm{z}$ was calculated for $\mathrm{C}_{34} \mathrm{H}_{34} \mathrm{~F}_{3} \mathrm{~N}_{2} \mathrm{O}_{4}[\mathrm{M}+\mathrm{H}]^{+} 591.2470$, and we found a ratio of 591.2471 ; RP-HPLC purity $96 \%$ at 254 $\mathrm{nm}, \mathrm{R}_{\mathrm{t}}=13.80 \mathrm{~min}$. Detailed experimental procedures, synthe- 
sis scheme, and NMR spectra regarding this compound can be found in the supporting information.

\section{Cell culture}

Huh7.5, HG23, and 293T cells were maintained in Dulbecco's modified Eagle's medium (DMEM, Hyclone, Logan, UT, USA) with $1 \%$ L-glutamine (Hyclone), $1 \%$ penicillin-streptomycin (Hyclone), 1\% non-essential amino acid (Hyclone), and $10 \%$ fetal bovine serum (JR Scientific, Woodland, CA, USA). All cells were cultured at $37^{\circ} \mathrm{C}$ in $5 \% \mathrm{CO}_{2}$. Huh7.5, $\mathrm{HG} 23$, and 293T cells were obtained from the American Type Culture Collection (ATCC) (Manassas, VA, USA).

\section{Plasmids}

To construct an HSF-1-expressing lentivirus, three plasmids were used: psPAX2, pMD2.G, and pBABE-HSF-FLAG WT, which were purchased from Addgene (Watertown, MA, USA) (Lesch et al., 2011). The psPAX2 vector was used to express the gag, pol, and env genes of lentivirus, while pMD2.G was used to express a VSV-G glycoprotein that allowed the virus to be transfected into unspecific host cells.

\section{Cell viability}

HG23 cells were plated onto a 96-well plate (Corning Inc., Corning, NY, USA) and treated with either DMSO or the compound of interest at designated concentrations. After 3 days, compound-induced cytotoxicity was measured by using EZCYTOX (10\% tetrazolium salt; DoGenBio, Seoul, Korea) reagent.

\section{Quantitative real-time RT-PCR}

HG23 cells were plated onto a 6-well plate (Costar) with either DMSO or the compound of interest $(10 \mu \mathrm{M})$. After 3 days, total cellular RNA was extracted using the RNeasy mini kit (Qiagen, Hilden, Germany) according to the manufacturer's protocol. The expression of subgenomic NV RNA and cellular RNA was quantified through quantitative real-time reverse-transcription polymerase chain reaction (qRT-PCR) analysis as previously described (Harmalkar et al., 2019). Each sample was normalized to the endogenous reference gene glyceraldehydes-3-phosphate dehydrogenase (gapdh). cDNA quantification was performed on the CFX384 real-time PCR detection system (Bio-Rad, Hercules, CA, USA). Primer sets used for qRT-PCR analysis were purchased from Macrogen (Seoul, Korea), and their sequences were as follows. FW-hNV, CGCTGGATGCGNTTCCATGA, and RV-hNV, CTTAGA CGCCATCATCATTTAC, for NV RNA quantitation; and FW-GAPDH, TGGTCTCCTCTGACTTCA, and RV-GAPDH, CGTTGTCATACCAGGAAATG, for GAPDH RNA quantitation.

\section{Western blot}

HG23 cells were plated onto a 6-well plate and supplemented with either DMSO or an increasing concentration of the compound of interest. Three days after incubation, wholecell extracts were prepared in RIPA buffer $(150 \mathrm{mM} \mathrm{NaCl}$, $1 \%$ Triton $\mathrm{X}-100,1 \%$ deoxycholic acid sodium salt, $0.1 \%$ sodium dodecyl sulfate, $50 \mathrm{mM}$ Tris- $\mathrm{HCl}, 2 \mathrm{mM}$ EDTA, $\mathrm{pH}$ 7.5; genDEPOT, Harris County, TX, USA) containing a cocktail of complete protease inhibitors (Roche, Basel, Switzerland) and quantitated by Bradford assay (Bio-Rad). Equal amounts of protein were electrophoresed on an SDS-polyacrylamide gel, transferred to a polyvinylidene difluoride membrane (Im-
mobilon-P; Millipore, Burlington, MA, USA), and probed with either a rabbit anti-neomycin phosphotransferase (1:500 dilution, ab33595, Abcam, Cambridge, UK) or rabbit anti-HSF-1 antibodies (1:1,000 dilution, D3L8I, Cell Signaling, Danvers, MA, USA).

\section{Immunofluorescence}

HG23 cells were cultured on cover glasses in a 24-well plate. After incubation, cells were treated with either $5 \mu \mathrm{M}$ or $10 \mu \mathrm{M}$ of AC-2288 for 24,48 , and $72 \mathrm{~h}$ and cultured at $37^{\circ} \mathrm{C}$ in $5 \% \mathrm{CO}_{2}$. After $72 \mathrm{~h}$, cells were washed with PBS three times and fixed with $4 \%$ formaldehyde for $15 \mathrm{~min}$. After washing with PBS three times, the cells were permeabilized with $0.1 \%$ Triton-X 100 for 2 min, then blocked in $2 \%$ FBS for $1 \mathrm{~h}$ at RT and incubated at $4{ }^{\circ} \mathrm{C}$ overnight with the following antibodies: anti-FLAG (Merck, F1804), anti-HSF-1 (Cell Signaling, D3L81), and anti-neomycin phosphotransferase 2 antibodies (Abcam, ab33595). After binding, Alexa 488-conjugated antimouse $\lg G$ or anti-rabbit lgG were reacted at RT for $40 \mathrm{~min}$. After nuclear staining with DAPI, the fluorescence signals were examined with a confocal microscope.

\section{HSF-1 downregulation}

HG23 cells and Huh7.5 cells were seeded into 6-well plates and transfected with the HSF-1-targeting siRNA called RNAiMAX (BIONEER, Daejeon, Korea) with final concentrations of 10, 50, and $100 \mathrm{nM}$ Lipofectamine 2000 (Invitrogen, Waltham, MA, USA) and incubated for $6 \mathrm{~h}$ at $37^{\circ} \mathrm{C}$. Then, cell culture media was changed. After that, incubation was carried out for $48 \mathrm{~h}$ at $37^{\circ} \mathrm{C}$.

\section{HSF-1 overexpression}

293T cells were seeded into a $10 \mathrm{~mL}$ plate and $24 \mathrm{~h}$ later, they were transfected with psPAX2, pMD2.G, and pBABEHSF-FLAG WT plasmids (Addgene) at a 1:1:1 ratio with Lipofectamin 2000 (Invitrogen). After $24 \mathrm{~h}$ of transfection, the media was changed and incubated for $48 \mathrm{~h}$ at $37^{\circ} \mathrm{C}$. The lentivirus present in the supernatant was collected and used for infecting target cells in the presence of polybrene in a 6-well plate. Then, cell culture medium was changed $24 \mathrm{~h}$ after infection, and infected target cells were incubated for $48 \mathrm{~h}$ at $37^{\circ} \mathrm{C}$.

\section{Statistical analysis}

Experimental results were expressed as the mean \pm the standard error of the mean (SEM) calculated as values measured by repeating experiments three or more times using Prism v5.0 (GraphPad, San Diego, CA, USA).

\section{RESULTS}

\section{Confirmation of the inhibitory activity of AC-1858 against NV RNA replication}

Before starting the elucidation of the antiviral mechanism of AC-1858 (Fig. 1A), we wished to confirm its antiviral activity using the NV replicon cells HG23. As shown in Fig. 1B, we were able to verify its dose-dependent antiviral activity $\left(\mathrm{EC}_{50}\right.$ $1.87 \mu \mathrm{M})$ against NV RNA replication with minimum cytotoxicity $\left(\mathrm{CC}_{50}>100 \mu \mathrm{M}\right)$. Based on these $\mathrm{EC}_{50}$ and $\mathrm{CC}_{50}$ values, its therapeutic index was calculated to be more than 53.48 . Subsequent reduction of viral protein expression due to its inhibitory effects on NV replication was further confirmed by 
A<smiles>C=Cc1c(OC)cc(/C=C/c2ccc(C(=O)N3CCN(Cc4ccc(C(F)(F)F)cc4)CC3)cc2)cc1OC</smiles>

C

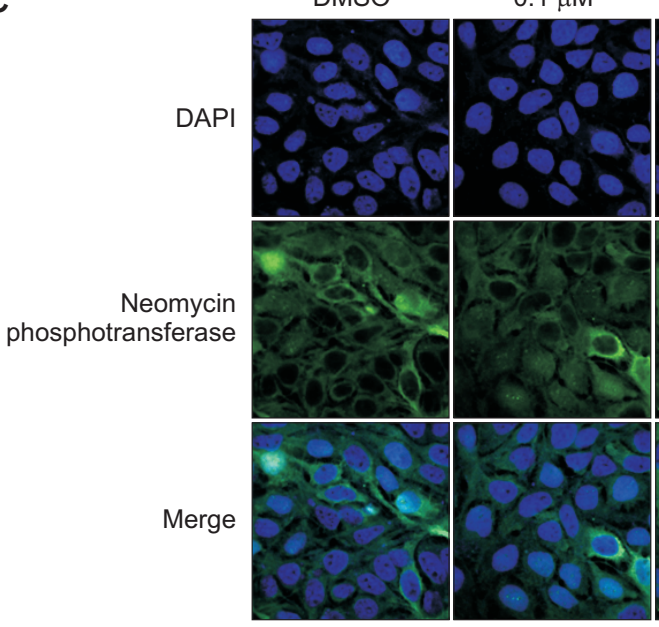

B

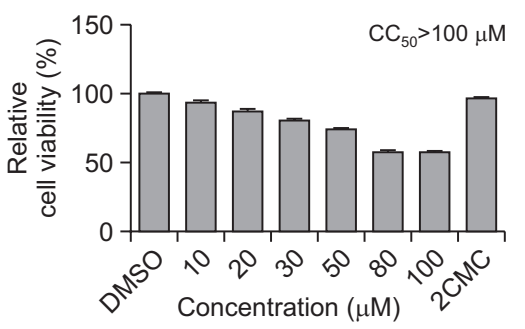

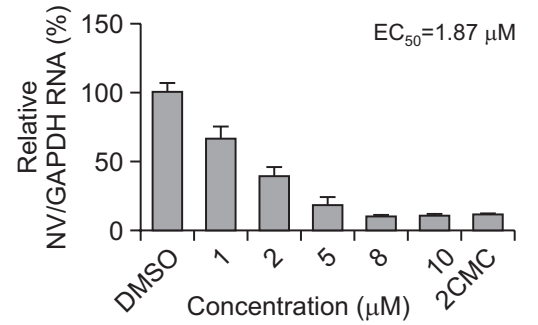

$5 \mu \mathrm{M}$

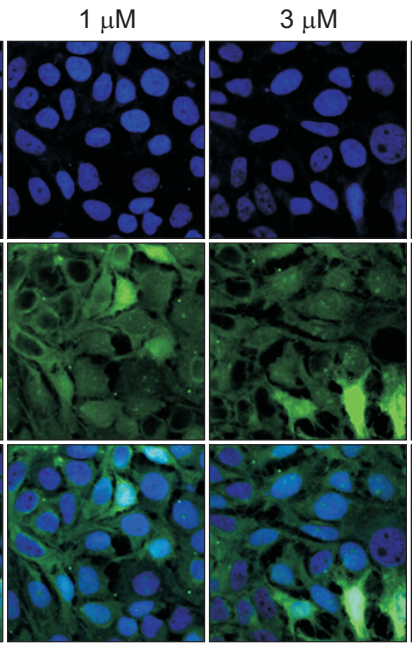

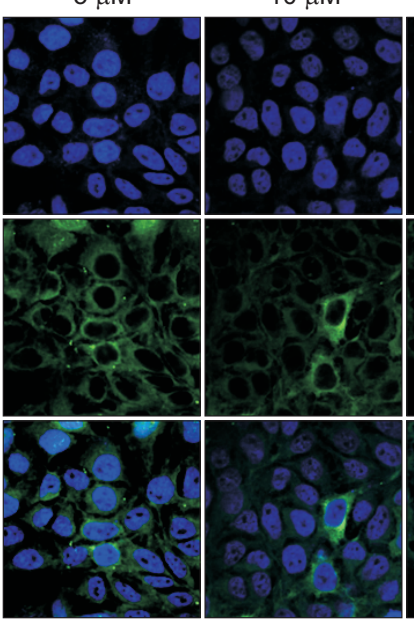

$2 \mathrm{CMC}$

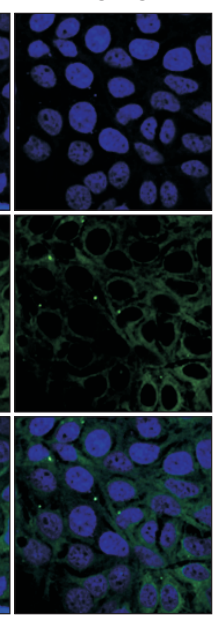

Fig. 1. Inhibition of NV RNA replication by AC-1858. (A) The chemical structure of a vinyl-stilbene compound, AC-1858, the most effective NV replication inhibitor. (B) HG23 cells were treated with increasing concentrations of AC-1858 for 72 h. Cell viability was measured by MTT assay. $\mathrm{CC}_{50}$ is the concentration required for $50 \%$ cytotoxicity. NV and GAPDH RNA levels were quantitated by qRT-PCR. 2'-C-methyl cytidine (2CMC), a viral polymerase inhibitor, was used as a positive control. $\mathrm{EC}_{50}$ is the concentration required for $50 \%$ inhibition of $\mathrm{NV}$ RNA replication. (C) An immunofluorescence assay was conducted to study the effects of AC-1858 on the expression of neomycin phosphotransferase. DAPI staining was used to indicate the locations of the nucleus in blue.

dose-dependent downregulation of the inserted neomycin phosphotransferase by immunofluorescence analysis (Fig. 1C). 2C-methyl cytidine (2CMC), which is a known nucleic acid analog inhibitor of NV RNA replication, was used as a positive control in both RT-PCR and immunofluorescence analyses (Fig. 1B, 1C).

\section{Inhibition of NV RNA replication by a structural analog AC-2288 via its cytoplasmic subcellular localization}

To visualize the subcellular locations of AC-1858 with its antiviral activity in action, we wished to synthesize a fluorescence-labeled version of AC-1858. For this goal, we synthesized the AC-2288 by introducing the carbonyl triple bond into the middle benzene ring of the original AC-1858 (Fig. 2A). Before starting a subcellular localization study, we decided to test the effects of the introduced carbonyl triple bond on antiviral activity and cell viability. As shown in Fig. 2B, AC-2288 turned out to possess more potent antiviral activity than the original AC-1858 (EC $0.99 \mu \mathrm{M})$. In addition, its cytotoxicity also seemed to be comparable to that of AC-1858 (Fig. 2B). Interestingly, we were also able to detect its strong autofluorescence without any conjugation with the intended fluorescence probe, such as FITC. When cells were treated with an increasing concentration of AC-2288, we were able to observe strong punctate fluorescence signals exclusively in the cyto- plasm in a dose- and time-dependent manner (Fig. 2C, 2D). This punctate localization pattern of AC-2288 suggested its potential presence in the ER membrane, which is a presumed site of NV RNA replication. In conclusion, these data indicate the ability of AC-2288 to penetrate the cell membrane and localize to the cytoplasmic area to exert its antiviral action against NV RNA replication.

\section{Induction of the phosphorylated form of HSF-1 by AC-1858}

Our previous PCR array analysis suggested the potential role of a host factor, HSF-1, in the pharmacological inhibition of NV RNA replication by AC-1858 (Harmalkar et al., 2019). In line with this result, we were able to confirm the dose-dependent induction of a phosphorylated form of HSF-1 by AC1858 treatment in HG23 cells. Interestingly, the amount of total HSF-1 proteins seemed to be unaffected by AC-1858 treatment (Fig. 3). Its parallel antiviral action was also confirmed by the concurrent reduced expression of neomycin phosphotransferase upon AC-1858 treatment (Fig. 3). Whereas 2CMC treatment diminished the levels of the phosphorylated form of HSF-1 with no effects on total HSF-1 proteins, suggesting its differential antiviral mode of action (Fig. 3). 
A<smiles>C=CCOc1cc(C(=O)N2CCN(Cc3ccc(C(F)(F)F)cc3)CC2)ccc1/C=C/c1cc(OC)c(C=C)c(OC)c1</smiles>

B

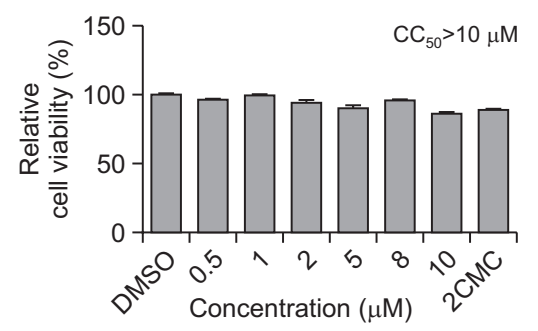

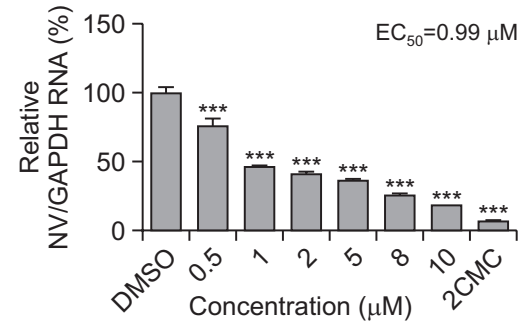

C

2CMC

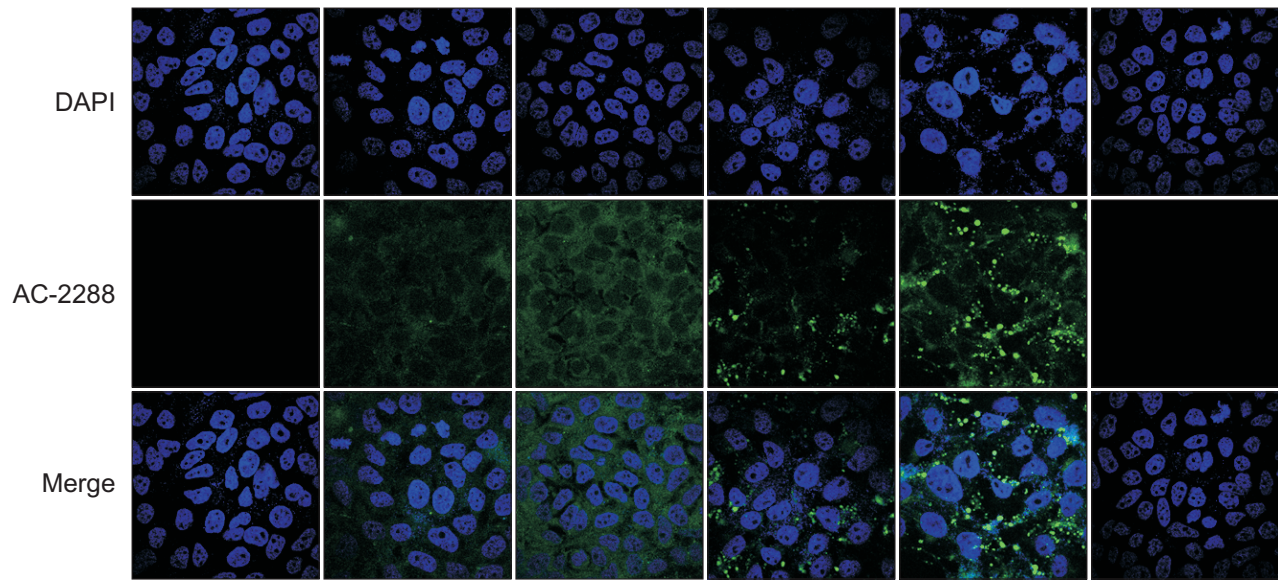

D

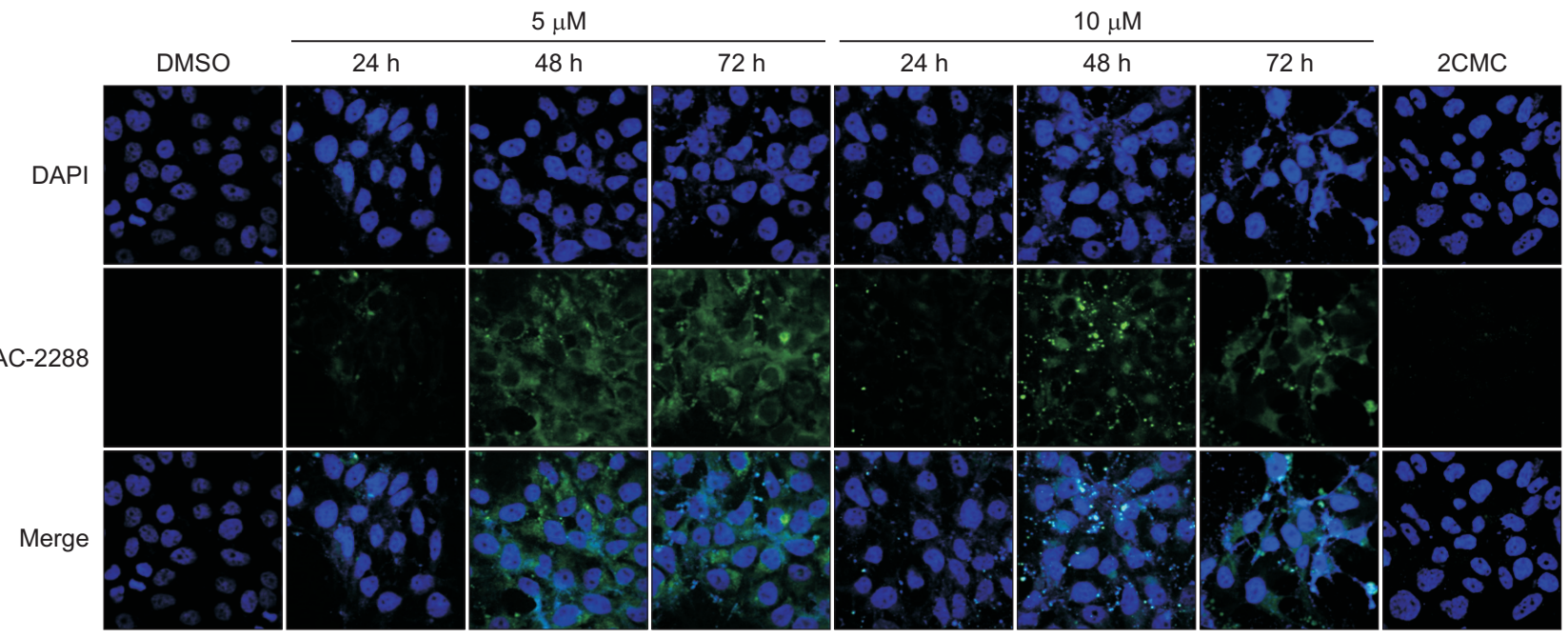

Fig. 2. Inhibition of the NV RNA replication by AC-2288 via its cytoplasmic localization. (A) The chemical structure of AC-2288, an analog compound of AC-1858. (B) HG23 cells were treated with increasing concentrations of AC-2288 for $72 \mathrm{~h}$. Cell viability was measured by MTT assay. $\mathrm{CC}_{50}$ is the concentration required for $50 \%$ cytotoxicity. NV and GAPDH RNA levels were quantitated by qRT-PCR. 2'-C-methyl cytidine (2CMC), a viral polymerase inhibitor, was used as a positive control. $\mathrm{EC}_{50}$ is the concentration required for $50 \%$ inhibition of NV RNA replication. (C) HG23 cells were treated with increasing concentrations of AC-2288 for $72 \mathrm{~h}$. Subcellular localization of AC-2288 was examined by a confocal microscope. DAPI staining was used to indicate the locations of the nucleus in blue. (D) HG23 cells were treated with either $5 \mu \mathrm{M}$ or $10 \mu \mathrm{M}$ of AC-2288. Subcellular localization of AC-2288 was examined at $24 \mathrm{~h}, 48 \mathrm{~h}$, and $72 \mathrm{~h}$ after treatment by a confocal microscope. DAPI staining was used to indicate the locations of the nucleus in blue. Calculated $p$ values, which were less than $0.0001^{* * *}$ when compared with a control, were considered statistically.

\section{Increased nuclear localization of HSF-1 by AC-1858}

HSF-1 exists as a monomer in the cytoplasm under unstressed conditions in cells. When cells are subjected to heat stress, it self-assembles into a trimer, which migrates to the nucleus to exert transactivation activity. Because we confirmed the induction of the phosphorylated form of HSF-1 by AC-1858 treatment, we wished to see if this AC-1858-induced induction of HSF-1 can lead to its increased nuclear localization. As we 
expected, we were able to observe its enhanced nuclear localization in a dose-dependent manner upon AC-1858 treatment (Fig. 4). Interestingly, the nuclear staining intensity of HSF-1 reached its plateau at $5 \mu \mathrm{M}$, after which it gradually decreased (Fig. 4). This data suggest the potential role of the enhanced nuclear localization of HSF-1 by AC-1858 in the inhibition of NV RNA replication.

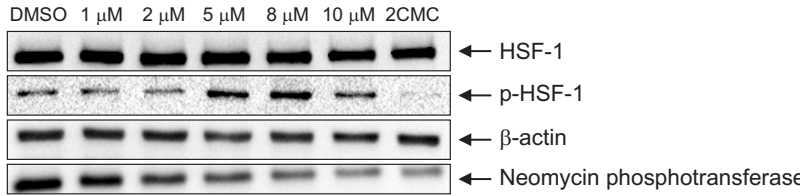

Fig. 3. Induction of the phosphorylated form of HSF-1 by AC1858. HG23 cells were treated with increasing concentrations of AC-1858 for 72 h. 2'-C-methyl cytidine (2CMC), a viral polymerase inhibitor, was used as a positive control. Expression levels of HSF1, phosphorylated HSF1, $\beta$-actin, and neomycin phosphotransferase proteins were analyzed by Western blotting with corresponding antibodies.

\section{Effects of downregulation and overexpression of HSF-1 on NV RNA replication}

After confirming the nuclear activation of HSF-1 by AC1858, we wanted to test the effects of the positive and negative genetic manipulation of HSF-1 expression on NV RNA replication. For the downregulation of the HSF-1 gene, we utilized three different kinds of commercially available HSF1-targeting siRNAs: siRNA-1, siRNA-2, and siRNA-3. While they did not have any deleterious effects on cell viability (Fig. $5 A$ ), they were able to suppress the expression of HSF-1, with siRNA-2 inducing the highest knockdown effect (Fig. 5B). In parallel, we also found that the reduced expression of HSF1 by siRNA-2 led to increased NV RNA replication (Fig. 5C). Conversely, when HSF-1 was overexpressed by lentiviral transduction (Fig. 6A), NV RNA replication progressively decreased with the increase in viral titer (Fig. 6B). This data suggest the potential negative role of HSF-1 in viral RNA genome replication during the $\mathrm{NV}$ life cycle.

\section{DISCUSSION}

We previously identified a vinyl-stilbene compound, AC-

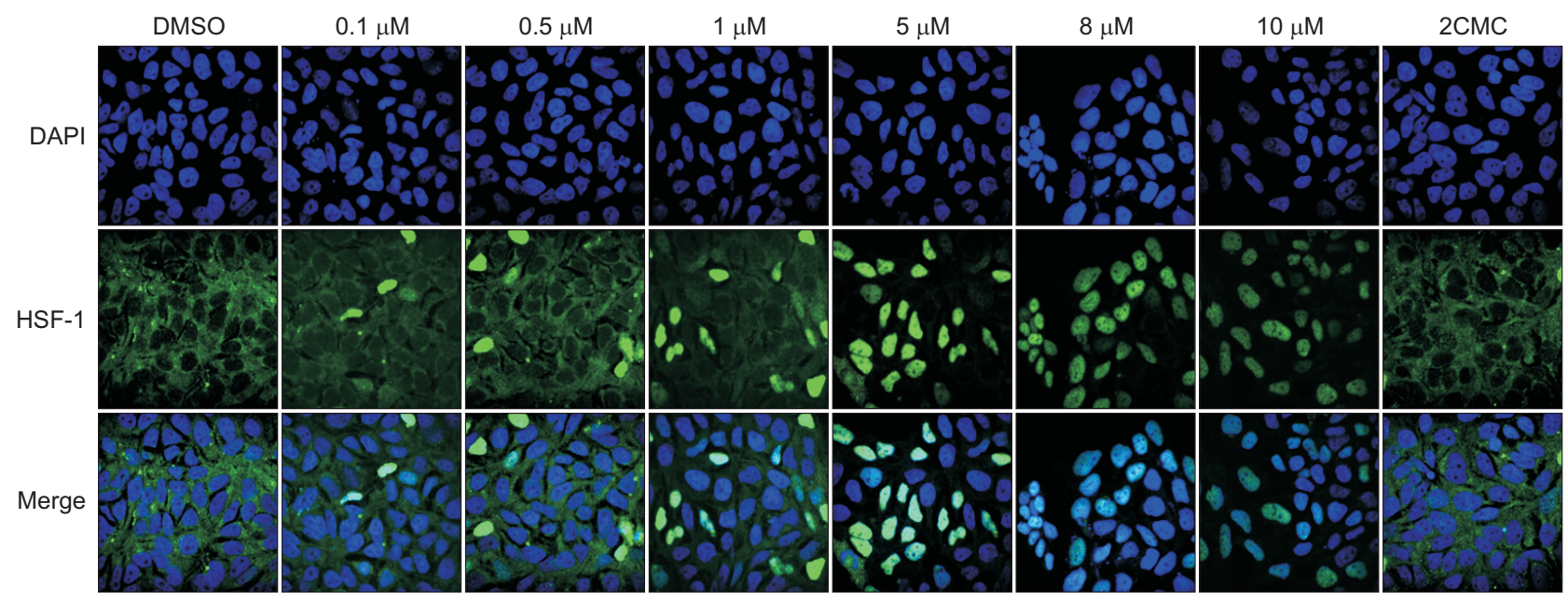

Fig. 4. Increased nuclear localization of HSF-1 by AC-1858. The effects of AC-1858 on the subcellular localization of the endogenous HSF1 protein were studied in HG23 cells by immunofluorescent analysis. DAPI staining was used to indicate the locations of the nucleus in blue.

A

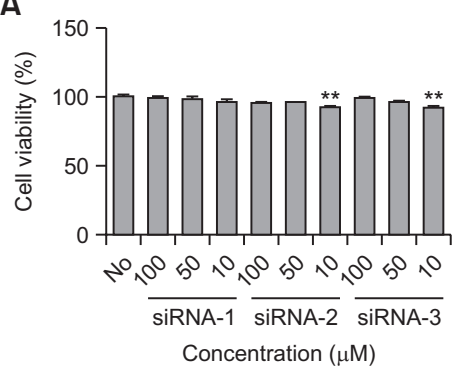

B

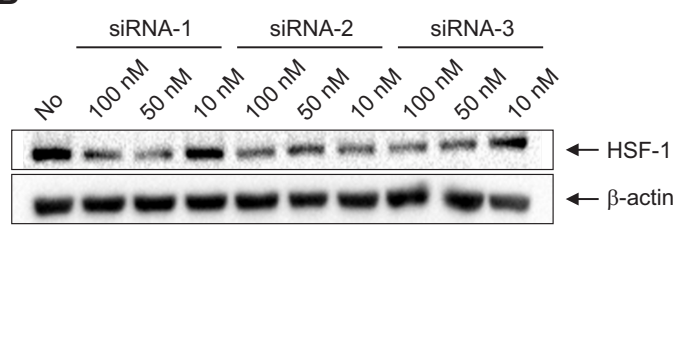

C

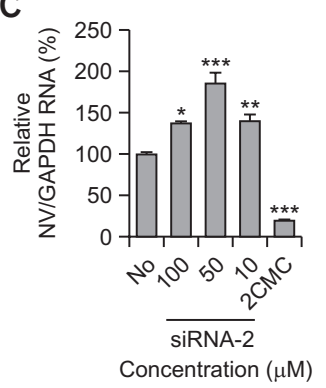

Fig. 5. Reduced expression of HSF-1 leads to increased NV RNA replication. HG23 cells (A) and Huh7.5 cells (B) were transfected with siRNA-1, -2 , and -3 , which target HSF-1 for degradation. (A) Cell viability was measured by MTT assay. (B) Expression levels of HSF-1 protein and $\beta$-actin proteins were analyzed after $48 \mathrm{~h}$ of siRNA transfection. (C) The NV and GAPDH RNA levels were quantitated by qRT-PCR in HG23 cells. 
A

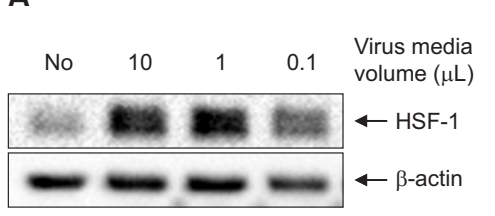

B

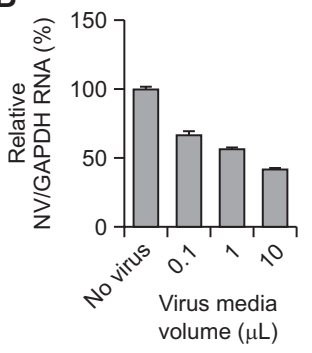

Fig. 6. Overexpression of HSF-1 leads to decreased NV RNA replication. HG23 cells were treated with $0.1,1$, and $10 \mu \mathrm{L}$ of HSF1-encoding lentivirus-containing media. (A) Expression levels of HSF-1 after lentiviral infection were quantitated by Western blot using an anti-HSF-1 antibody. (B) The NV and GAPDH RNA levels were quantitated by qRT-PCR in HG23 cells.

1858, as a potent NV replication inhibitor (Harmalkar et al., 2019). In regards to its potential antiviral mechanism of action, we suggested a host factor, HSF-1, is a critical player for AC-1858-mediated inhibition of NV RNA replication (Harmalkar et al., 2019). In this study, we sought to further elucidate the molecular mechanisms underlying the antiviral action of AC-1858 against NV RNA replication through positive modulation of HSF-1 function. To achieve this goal, we first confirmed the inhibition of NV RNA replication by the structural analog AC-2288 with its exclusive cytoplasmic subcellular localization (Fig. 1, 2). Then, we further validated the induction of the phosphorylated form of HSF-1 and the increased nuclear localization of HSF-1 by AC-1858 (Fig. 3, 4). Finally, we verified the positive and negative impact of siRNA-mediated downregulation and lentivirus-mediated overexpression of HSF-1 on NV RNA replication, respectively (Fig. 5, 6). Based on these findings, we suggest HSF-1 has a restrictive role in overall viral RNA genome replication.

NV builds its replication complex by expressing nonstructural viral proteins for the synthesis of the viral RNA genome on the ER membrane. Therefore, the correct delivery of a potential antiviral candidate to the ER membrane increases the possibility of the successful execution of its antiviral action against the NV replication complex. To conduct a microscopic study of the subcellular locations of AC-1858, we needed to conjugate a fluorescent probe to AC-1858 for visualization. For this, we added the carbonyl triple bond to the middle benzene ring of AC-1858 for fluorescence conjugation (Fig. $2 A)$. Since the addition of an extra structure onto the original backbone of AC-1858 might have a deleterious effect on both cell viability and antiviral activity, we wished to exclude this possibility. Luckily, the modification of the middle benzene ring did not produce any detrimental effects on cell viability and antiviral activity (Fig. 2B). Unexpectedly, this carbonyl triplebond-added compound, AC-2288 turned out to be auto-fluorescent, which alleviated the need to add an extra fluorescent dye, such as FITC, to this compound. In addition, its autofluorescence-induced punctate localization patterns after plasma membrane penetration strongly suggested the characteristic ER-specific subcellular localization of AC-1858 with its maximal localization visible around 72-h post-treatment (Fig. 2C, 2D). Based on these data, we hypothesized that AC-1858 has an ER-targeting property that could be harnessed to increase its potential antiviral activity against NV RNA replication.

HSF-1 generally exists as a single entity in the cytoplasm, but when the cell is subjected to heat stress, it is phosphorylated by various kinases, converted into a trimer, and moves into the nucleus in an activated state, finally acting as a transcription activator to stimulate the transcription of a variety of heat shock protein (HSP) genes. Interestingly, one of the HSP genes, HSP90, was shown to affect the stability of the NV capsid protein (Vashist et al., 2015). Based on the data presented in Fig. 3, AC-1858 seems to be able to transform the inactive state of HSF-1 into the active state by increasing the phosphorylated form of HSF-1. Treatment of AC-1858 might activate the enzymatic activity of a certain unknown host kinase that is responsible for the subsequent enhancement of HSF-1 phosphorylation. In a future study, we will focus on the identification of a panel of host kinases that might be pharmacologically activated by AC-1858 treatment.

Increased nuclear import of HSF-1 by AC-1858 is supposed to increase the transcription of HSF-1 target genes like HSPs. According to this model, these downstream effector HSPs should be directly involved in the induction of the anti-NV replication activity of AC-1858. However, a number of previous studies seem to be discordant with this hypothesis. For example, in previous studies on murine NV, which is closely related to human NV, HSP70 and HSP90 produced by HSF-1 played a positive role in NV replication (Ma et al., 2020). In agreement with this finding, another group also reported the negative impacts inhibiting the HSP90 activity on NV replication in cell culture (Vashist et al., 2015). In this study, they also found that small-molecule-mediated inhibition of HSP90 activity led to the potent suppression of NV RNA replication. To resolve this discrepancy, we also examined the mRNA levels of HSP70 and HSP90 in HG32 cells after AC-1858 treatment. To our surprise, we found decreased mRNA expression of both HSP70 and HSP90 in HG32 cells after AC-1858 treatment, despite the presence of the enhanced phosphorylated form of HSF-1 and its increased nuclear import (data not shown). This was in sharp contrast to our expectations, considering the transactivation function of HSF-1 in the transcriptional induction of its target genes, such as HSP70 and HSP90. Although the reduced expression of both HSP70 and HSP90 by AC-1858 treatment might lead to the inhibition of NV RNA replication, as suggested by previous results (Ma et al., 2020), this model needs to be re-examined in a more experimentally defined manner. Alternatively, HSF-1-activated target genes other than HSP70 and HSP90 may play roles in the AC1858-mediated inhibition of NV RNA replication. The downand up-regulation of HSF-1 clearly demonstrated the negative role played by HSF-1 in the overall modulation of NV RNA genome replication (Fig. 5,6). Further research is needed to provide a better understanding of the pharmacological actions of AC-1858 in the context of NV RNA replication.

In conclusion, we elucidated the restrictive role of HSF-1 in the pharmacological modulation of NV RNA replication by AC1858. Based on this study, endeavors to design a new pharmacophore with better HSF-1-inducer activity should yield a fruitful host-modulating antiviral strategy in the development of a new class of anti-NV therapeutics in the future.

\section{ACKNOWLEDGMENTS}

This research was supported by the Basic Science Re- 
search Program through the National Research Foundation of Korea (NRF) funded by the Ministry of Education (NRF-2019R1F1A1058628). This work was also supported by the National Research Foundation of Korea (NRF) grant funded by the Korean government (MSIT) (No. NRF2018R1A5A2023127).

\section{REFERENCES}

Atmar, R. L., Baehner, F., Cramer, J. P., Lloyd, E., Sherwood, J., Borkowski, A. and Mendelman, P. M.; NOR-201 Study Group (2019) Persistence of antibodies to 2 virus-like particle norovirus vaccine candidate formulations in healthy adults: 1-year follow-up with memory probe vaccination. J. Infect. Dis. 220, 603-614.

Atmar, R. L. and Estes, M. K. (2006) The epidemiologic and clinical importance of norovirus infection. Gastroenterol. Clin. North Am. 35, 275-290.

Bok, K. and Green, K. Y. (2012) Norovirus gastroenteritis in immunocompromised patients. N. Engl. J. Med. 367, 2126-2132.

Chang, K. O., Sosnovtsev, S. V., Belliot, G., King, A. D. and Green, K. Y. (2006) Stable expression of a Norwalk virus RNA replicon in a human hepatoma cell line. Virology 353, 463-473.

de Graaf, M., van Beek, J. and Koopmans, M. P. (2016) Human norovirus transmission and evolution in a changing world. Nat. Rev. Microbiol. 14, 421-433.

Glass, R. I., Parashar, U. D. and Estes, M. K. (2009) Norovirus gastroenteritis. N. Engl. J. Med. 361, 1776-1785.

Hardy, M. E. (2005) Norovirus protein structure and function. FEMS Microbiol. Lett. 253, 1-8.

Harmalkar, D. S., Lee, S. J., Lu, Q., Kim, M. I., Park, J., Lee, H., Park,
M., Lee, A., Lee, C. and Lee, K. (2019) Identification of novel nonnucleoside vinyl-stilbene analogs as potent norovirus replication inhibitors with a potential host-targeting mechanism. Eur. J. Med. Chem. 184, 111733

Lee, H. and Ko, G. (2016) Antiviral effect of vitamin A on norovirus infection via modulation of the gut microbiome. Sci. Rep. 6, 25835.

Lesch, H., Laitinen, A., Peixoto, C., Vicente, T., Makkonen, K., Laitinen, L., Pikkarainen, J., Samaranayake, H., Alves, P., Carrondo, M., Ylä-Herttuala, S. and Airenne, K. J. (2011) Production and purification of lentiviral vectors generated in 293T suspension cells with baculoviral vectors. Gene Ther. 18, 531-538.

Ma, C., Zhang, X., You, J., Dong, M., Yun, S. and Liu, J. (2020) Effect of heat shock on murine norovirus replication in RAW264.7 cells. Microb. Pathog. 142, 104102.

Morillo, S. G. and Timenetsky, M. d. C. S. T. (2011) Norovirus: an overview. Rev. Assoc. Med. Bras. 57, 453-458.

Thorne, L. G. and Goodfellow, I. G. (2014) Norovirus gene expression and replication. J. Gen. Virol. 95, 278-291.

Turcios, R. M., Widdowson, M. A., Sulka, A. C., Mead, P. S. and Glass, R. I. (2006) Reevaluation of epidemiological criteria for identifying outbreaks of acute gastroenteritis due to norovirus: United States, 1998-2000. Clin. Infect. Dis. 42, 964-969.

Vashist, S., Urena, L., Gonzalez-Hernandez, M. B., Choi, J., de Rougemont, A., Rocha-Pereira, J., Neyts, J., Hwang, S., Wobus, C. E. and Goodfellow, I. (2015) Molecular chaperone Hsp90 is a therapeutic target for noroviruses. J. Virol. 89, 6352-6363.

Vinje, J., Green, J., Lewis, D., Gallimore, C., Brown, D. and Koopmans, M. (2000) Genetic polymorphism across regions of the three open reading frames of "Norwalk-like viruses". Arch. Virol. 145, 223-241.

Wobus, C. E., Thackray, L. B. and Virgin, H. W., IV (2006) Murine norovirus: a model system to study norovirus biology and pathogenesis. J. Virol. 80, 5104-5112. 